\title{
BMJ Open Determinants of treatment non- attendance among those referred to primary mental health care services in Western Sydney, Australia: a retrospective cohort study
}

\author{
Sithum Munasinghe (D , ${ }^{1}$ Andrew Page, ${ }^{1}$ Haider Mannan, ${ }^{1}$ Shahana Ferdousi, ${ }^{2}$ \\ Brendan Peek ${ }^{3}$
}

To cite: Munasinghe S, Page A, Mannan $\mathrm{H}$, et al. Determinants of treatment non-attendance among those referred to primary mental health care services in Western Sydney, Australia: a retrospective cohort study. BMJ Open 2020;10:e039858. doi:10.1136/ bmjopen-2020-039858

- Prepublication history and additional material for this paper is available online. To view these files, please visit the journal online (http://dx.doi.org/10. 1136/bmjopen-2020-039858)

Received 28 April 2020

Revised 05 September 2020

Accepted 11 September 2020

A Check for updates

(c) Author(s) (or their employer(s)) 2020. Re-use permitted under CC BY-NC. No commercial re-use. See rights and permissions. Published by BMJ.

${ }^{1}$ Translational Health Research Institute, Western Sydney University, Penrith, New South Wales, Australia

${ }^{2}$ Western Sydney Primary Health Network, Blacktown, New South Wales, Australia

${ }^{3}$ Royal Australasian College of Dental Surgeons, Sydney, New South Wales, Australia

Correspondence to Sithum Munasinghe s.munasinghe@westernsydney. edu.au

\section{ABSTRACT}

Objectives Recommendations of the recent mental health reforms provided an opportunity to implement regional approaches to service provision through Primary Health Networks. This study is designed to identify the determinants of sociodemographic, diagnostic and referral-level factors and first treatment session non-attendance among those referred to primary mental health care (PMHC) services in Western Sydney, Australia.

Design This study used routinely collected retrospective PMHC data between July 2016 and December 2018.

Setting The study was based on a geographical catchment that covers four local government areas of Blacktown, Parramatta, Cumberland and Hills Shire in Western Sydney, Australia.

Participants All individuals 5 years of age or older referred to PMHC services.

Primary outcome measure First treatment session nonattendance, following a referral to receive psychological treatments.

Results There were 9158 referrals received for 8031 clients, with 1769 (19.32\%) referrals resulting in nonattendance to the first treatment session. Those with younger age (ORs ranging from 1.63 to 1.92), substance use $(\mathrm{OR}=1.55,95 \% \mathrm{Cl} 1.17$ to 2.06), poor English proficiency $(\mathrm{OR}=1.64,95 \% \mathrm{Cl} 1.23$ to 2.20$)$, lower socioeconomic status ( $\mathrm{OR}=1.57,95 \% \mathrm{Cl} 1.34$ to 1.83$)$, psychotropic medication use $(0 \mathrm{R}=1.20,95 \% \mathrm{Cl} 1.06$ to 1.36), and a referral by a social worker $(\mathrm{OR}=2.04,95 \%$ $\mathrm{Cl} 1.36$ to 3.05$)$, allied health $(\mathrm{OR}=1.49,95 \% \mathrm{Cl} 1.03$ to 2.16) or other professional $(0 \mathrm{R}=1.72,95 \% \mathrm{Cl} 1.30$ to 2.29$)$ were associated with a higher likelihood of first treatment session non-attendance. Those with a risk of suicide, who mainly speak a language other than English, and a previous use of PMHC services were more likely to attend their first treatment session.

Conclusion Youth-specific treatment approaches, behavioural engagement strategies, facilitation of transport services for those live in deprived regions and improvements in capacity for mental health training among allied health professionals are areas of focus for primary care service and policy responses.

\section{Strength and limitations of the study}

- This study investigates the determinants of first treatment session non-attendance to primary mental health care services. This is of current relevance in Australia given recent national policy reforms to organise regionally specific approaches to mental health service provision.

- The study has a large sample size, and multiple imputation techniques were also employed to maximise the use of data.

- Age-specific analyses were conducted to investigate differences in the association between determinants of treatment non-attendance among younger age groups (age $\leq 24)$ and older age groups (age $>24$ ).

- There may be important covariates that were not captured in the current study and which may also explain the treatment non-attendance.

- There may be a group of patients who did not attend these free or low-cost services, and may have accessed other fee for service mental health services.

\section{INTRODUCTION}

Non-attendance to treatments following a referral is a common phenomenon in the primary health care sector, and is more likely to occur in mental health care settings compared with other primary health care contexts. ${ }^{1}$ Treatment non-attendance can potentially affect deterioration in symptoms, quality of life $\mathrm{e}^{1}$ and other mental health outcomes. For example, subsequent hospital admission rates have been shown to be higher among those who did not attend their first appointment for psychiatric outpatient treatment following referral from a primary care setting, ${ }^{2}$ and a recent study indicated that interventions to re-engage those lost to mental health services had the greatest potential impact on reducing the incidence of suicide. ${ }^{3}$ In addition, treatment non-attendance is also 
associated with additional work load and administrative burden in following-up patients and managing service attendance, ${ }^{4}$ potentially decreasing the expected costeffectiveness of services.

Treatment non-attendance can refer to those who never initiate treatment sessions for a given referral (first treatment non-attendance) and those who disengage with, or terminate, treatments (treatment dropout) against a service provider's recommendation. Previous studies have demonstrated differential patterns in these two forms of treatment non-attendance for patient demographic, clinical and service level characteristics. ${ }^{5}$ There is no universal definition of treatment drop out or disengagement used. ${ }^{7}$ Some studies have used number of sessions to define treatment dropout, but this can be problematic due to the methodological differences in terms of patient demographics, severity of mental health conditions and service-level characteristics, and also the use of different categories of session frequency between studies. ${ }^{7}$ Information on number of treatment sessions can be difficult to interpret in routinely collected primary care data, where a non-attendance could reflect treatment drop out, referral to other services, or coding errors. It could also reflect treatment completion as previous research evidence indicates psychotherapy dropout even after the first session may also reflect improvements in a given mental health conditions. ${ }^{8}$ Hence, the current study focusses on the determinants of first treatment non-attendance.

Previous research has indicated that sociodemographic factors such as younger age, race, being unmarried and lower socioeconomic status (SES) have been associated with a higher risk of first treatment session non-attendance. ${ }^{159-12}$ Additionally, substance use as a main diagnosis (or as a comorbid condition), personality disorder, risk of suicide and previous mental health service use have also been associated with nonattendance. ${ }^{151013}$ Other referral-level factors associated with non-attendance also include referrer profession, poor communication between referrer and patient, and waiting time to first treatment appointment. ${ }^{161415}$

This question is of current relevance in Australia given recent national policy reforms, which have aimed to provide more appropriate and regionally specific mental health services through Primary Health Networks (PHNs). ${ }^{16}$ The current study is based on primary mental health care services (PMHC) in Western Sydney (Australia), a sociodemographically heterogeneous geographic region comprising a high proportion of culturally and linguistically diverse communities. ${ }^{17}$ Although there has been previous research conducted on first treatment session non-attendance, the extent to which recommendations in these studies are applicable to the Western Sydney population catchment is uncertain due to the heterogeneity of population backgrounds and differences in mental health services provided in a primary care setting, and changes over time in mental health policies and services. The aim of the current study is to investigate the sociodemographic, clinical and referral-level determinants of first treatment session nonattendance among those referred to the PMHC services in Western Sydney (Australia). Findings can be used to improve treatment engagement among population subgroups that are more likely to not initiate treatments, despite a referral to PMHC services.

\section{METHODS}

\section{Study catchment}

This study was based on the Western Sydney PHN population catchment that covers four local government areas of Blacktown, Parramatta, Cumberland and Hills Shire in Western Sydney, Australia. The estimated population in this geographical setting was approximately 950000 in $2016 .{ }^{18}$ In addition, the Western Sydney PHN catchment comprises the highest proportion $(39.4 \%)$ of people born in predominantly non-English speaking countries compared with all other 30 PHN catchment areas in Australia. ${ }^{17}$

\section{Primary mental health care services}

The PMHC services considered in this study are federal government initiatives established to support those who otherwise have no, or limited, access to mental health services, established under the Better Outcomes in Mental Health Care Programme in 2001 to facilitate mental health service access. ${ }^{19}$ As part of this programme, general practitioners (GPs) were provided incentives to work collaboratively with mental health professionals under the Access To Allied Psychological Services (ATAPS) and to refer people with mental disorders to receive psychological therapies where appropriate. While there is no limit to the number of sessions per referral for individuals, under this scheme now, clients are required to visit their GP for a mental health review after every six sessions, and can extend for another six sessions based on the client's specific circumstances

These services were first commissioned by Divisions of General Practice and were transitioned to Medicare Locals in 2011, ${ }^{20}$ and then to PHNs in July 2016 following the recommendations of a major mental health review conducted in Australia in 2014. ${ }^{16}$ These recommendations proposed a more regional approach to mental health and suicide prevention services. Under these reforms, PMHC services are designed to deliver mental health support under eight main focus areas including (1) psychological therapy; (2) low intensity psychological interventions; (3) clinical care coordination; (4) complex care packages; (5) child and youth specific mental health services; (6) Indigenous-specific mental health services; (7) other and (8) psychosocial support services.

\section{Participants}

This study included routinely collected de-identified data of those age $\geq 5$ years, referred to PMHC services between July 2016 and December 2018. The majority of the referrals were for psychological therapy (7987, 72.6\%), 
followed by low intensity psychological interventions $(1758,15.5 \%)$ and child and youth specific mental health services $(650,7.1 \%)$. However, referrals to low intensity psychological interventions $(1758,15.5 \%)$, clinical care coordination $(60,0.53 \%)$ and psychosocial support (18, $0.16 \%$ ) were not included due to the inconsistent data reporting.

\section{Patient and public involvement}

Patients and the public were not involved in the study or study design, except for their contribution to the health records. Because this study used routinely collected de-identified data, it was not feasible to involve patients or the public in the design or conduct of the study.

\section{Data sources}

Data for PMHC services were extracted from the PMHC national Minimum Data Set (MDS) and ATAPS MDS. The PMHC MDS was developed based on the previous ATAPS MDS data architecture, but collects a broader range of information than the previous ATAPS MDS. The PMHC MDS is designed to collect mental health data from treatment providers commissioned by PHNs to provide primary care services and provides the basis for PHNs and the Commonwealth Department of Health to monitor the quantity and quality of mental health treatment delivery. Treatment providers are required to enter or upload data into the PMHC MDS central web portal. The PMHC MDS comprises items relating to patients' sociodemographic and socioeconomic information, mental health referral and diagnosis information, service session information and practitioner characteristics. Since the establishment of the PMHC MDS, all ATAPS MDS data were incorporated into the current PMHC MDS in July 2017. The current study is based on data extracted from both ATAPS and PMHC MDSs for the period between July 2016 and December 2018. In addition to the PMHC data, this study also used the Index of Relative Socio-economic Advantage and Disadvantage for the census year $2016^{21}$ to derive area-level SES based on the postcodes of those referred to PMHC services.

\section{Study variables}

The outcome variable of this study was first treatment nonattendance, a binary variable classified as either 'did not attend' or 'attended'. If any referral received for psychological treatments ended without at least one treatment session, then that particular referral was classified as 'did not attend'. A series of time variant (some clients were referred more than once during the period of study) and time invariant sociodemographic, diagnostic and referral level variables were investigated as determinants of treatment non-attendance, and were based on available data items. All the variables except gender may be time dependent for those who were referred more than once during the study period.

Sociodemographic variables included gender (male, female), age group $(5-11,12-24,25-44,45-64, \geq 65)$, main language spoken at home (English, other), proficiency of English (not at all/not well, well, very well), arealevel SES (population quintiles from least deprived to most deprived), marital status (never married, divorced, separated, widowed, married (registered or de facto)) and employment status (full time, part time, not in the labour force).

Diagnostic factors included presenting diagnosis (anxiety disorders, depressive disorders, both anxiety and depressive disorders, substance use disorders, other) and suicidal risk (yes, no), classified as 'yes' if the presentation followed a suicide attempt or with suicide ideation.

Other referral level variables included referrer profession (GP, psychologist, mental health nurse, social worker, psychiatrist, paediatrician, other), previous history of PMHC service use (irrespective of whether individuals completed treatments or not for a given episode/s during the past 14 years of data availability) (yes, no) or use of any mental health medication during the time of referral (yes, no). Mental health medication included use of any antipsychotics, anxiolytics, hypnotics and sedatives, antidepressants, or psychostimulants and nootropics. The generic term 'PMHC' is used in the current study hereafter to refer to PMHC services.

\section{Statistical analysis}

Descriptive analyses presented counts and percentages for each study factor. Generalized estimating equations (GEEs) were employed with a logit link and exchangeable correlation structure to model the association between time variant and invariant variables and first session non-attendance. Univariate and multivariate analyses adjusting for each of the covariates were conducted and ORs with 95\% CIs were presented. Interaction effects between covariates were also examined. Age-specific analyses were also conducted by stratifying models to investigate the associations between study factors and first treatment session non-attendance among younger ( $\leq 24$ years) and older ( $>24$ years) age cohorts. Marital status and employment status were available as variables in the database since July 2017 (not included in ATAPS MDS) and the effect sizes of these two variables on the outcome were derived from the same GEE models for the period July 2017 to December 2018. Marital status and employment status were not included as covariates in models of those aged $\leq 24$ years.

Missing data were present in some of the covariates in the data set. In order to maximise the use of available information, we employed the Multivariate Imputation by Chained Equation method (MICE) using the MICE package in $\mathrm{R},{ }^{22}$ and generated 20 separate imputations using 20 cycles to impute a single missing value of a variable in order to minimise the uncertainty around estimates. Complete case multivariable analyses were based on $76.9 \%$ of the total sample for all ages, $77.1 \%$ for the younger age group ( $\leq 24$ years), and $76.8 \%$ for the older age group ( $>24$ years). All statistical analyses were 
Table 1 Sociodemographic, diagnostic and referral information

\begin{tabular}{|c|c|c|c|}
\hline Characteristics & $\begin{array}{l}\text { Attended } \\
\mathrm{N}=7389(80.68 \%)\end{array}$ & $\begin{array}{l}\text { Did not attend } \\
\mathrm{N}=1769(19.32 \%)\end{array}$ & $\begin{array}{l}\text { Total } \\
\mathrm{N}=9158(100 \%)\end{array}$ \\
\hline \multicolumn{4}{|c|}{ Gender (data missing for 20 (0.22\%) referrals) } \\
\hline Male & $2904(39.38)$ & $734(41.61)$ & $3638(39.81)$ \\
\hline Female & $4470(60.62)$ & $1030(58.39)$ & $5500(60.19)$ \\
\hline \multicolumn{4}{|l|}{ Age $^{*}$} \\
\hline$\geq 65$ & $508(6.88)$ & $80(4.52)$ & $588(6.42)$ \\
\hline $25-44$ & $2353(31.84)$ & $601(33.97)$ & $2954(32.26)$ \\
\hline $12-24$ & $1733(23.45)$ & $498(28.15)$ & $2231(24.36)$ \\
\hline$\leq 11$ & $1003(13.57)$ & $275(15.55)$ & $1278(13.96)$ \\
\hline \multicolumn{4}{|c|}{ Proficiency of English (data missing for 395 (4.31\%) referrals) } \\
\hline \multicolumn{4}{|c|}{ Main Language at home (data missing for 321 (3.51\%) referrals) } \\
\hline English & $6586(92.42)$ & $1622(94.8)$ & $8208(92.88)$ \\
\hline Other & $540(7.58)$ & $89(5.2)$ & $629(7.12)$ \\
\hline \multicolumn{4}{|c|}{ Socioeconomic statust (data missing for $73(0.8 \%)$ referrals) } \\
\hline 5 (least deprived) & $1707(23.27)$ & $330(18.86)$ & $2037(22.42)$ \\
\hline 4 & $1752(23.89)$ & $386(22.06)$ & $2138(23.53)$ \\
\hline 3 & $1650(22.49)$ & $408(23.31)$ & $2058(22.65)$ \\
\hline 2 & $488(6.65)$ & $65(3.71)$ & $553(6.09)$ \\
\hline 1 (most deprived) & $1738(23.69)$ & $561(32.06)$ & $2299(25.31)$ \\
\hline \multicolumn{4}{|c|}{ Marital statusł (data missing for 1326 (27.95\%) referrals) } \\
\hline \multicolumn{4}{|c|}{ Employment statusł (data missing for 1429 (30.12\%) referrals) } \\
\hline Full time & $306(11.25)$ & $85(14.29)$ & $391(11.79)$ \\
\hline Part time & $420(15.44)$ & $78(13.11)$ & $498(15.02)$ \\
\hline Not in the labour force & 1994 (73.31) & $432(72.61)$ & $2426(73.18)$ \\
\hline \multicolumn{4}{|c|}{ Diagnosis (data missing for 269 (2.94\%) referrals) } \\
\hline Anxiety disorders & $1550(21.59)$ & $387(22.63)$ & $1937(21.79)$ \\
\hline Affective (mood) disorders & $1863(25.95)$ & $442(25.85)$ & $2305(25.93)$ \\
\hline Anxiety and affective disorders & $2838(39.53)$ & $625(36.55)$ & $3463(38.96)$ \\
\hline Substance use disorders & $234(3.26)$ & $87(5.09)$ & $321(3.61)$ \\
\hline Other & $694(9.67)$ & $169(9.88)$ & $863(9.71)$ \\
\hline \multicolumn{4}{|c|}{ Suicide risk (data missing for 242 (2.64\%) referrals) } \\
\hline No & $6185(85.68)$ & $1491(87.86)$ & $7676(86.09)$ \\
\hline Yes & $1034(14.32)$ & $206(12.14)$ & $1240(13.91)$ \\
\hline \multicolumn{4}{|c|}{ Referrer type (data missing for $54(0.59 \%)$ referrals) } \\
\hline General practitioner & $6668(90.8)$ & 1520 (86.36) & 8188 (89.94) \\
\hline Psychologist & $55(0.75)$ & $18(1.02)$ & $73(0.8)$ \\
\hline Mental health nurse & $131(1.78)$ & $42(2.39)$ & $173(1.9)$ \\
\hline Social worker & $79(1.08)$ & $37(2.1)$ & $116(1.27)$ \\
\hline
\end{tabular}


Table 1 Continued

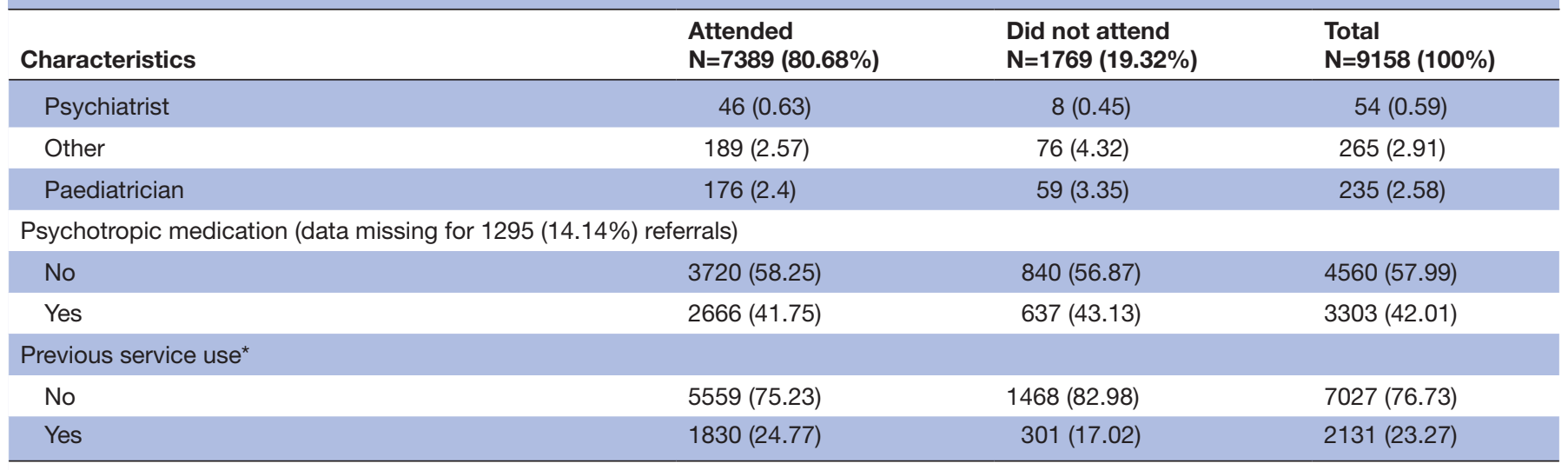

$\mathrm{N}$-number of referrals.

Those who attended versus did not attend the first treatment session.

*No missing values recorded.

†Area socioeconomic status, based on Index of Relative Socio-economic Advantage and Disadvantage.

¥Counts and denominator included those referred between July 2017 and December 2018 and age $\geq 18$.

conducted using Stata V.14.0 (Stata Crop, 4905 Lakeway Dive, College Station, TX77845, USA) and R V.3.6.0.

\section{RESULTS}

There were 9158 referrals received for 8031 patients for the period of July 2016 to December 2018. Of these referrals, $1769(19.3 \%)$ referrals did not result in any subsequent service session attendance. Nearly $60 \%$ of the referrals were for females, more than two-thirds for those aged $\leq 44$ years, (table 1 ). Nearly $40 \%$ of the referrals for those were never married and $73 \%$ for those were not in the labour force. Depression or anxiety was the main reasons for referrals to the PMHC services. Over $40 \%$ of those referred used psychotropic medication at the time of referral and approximately $14 \%$ of those presented with suicide risk (table 1). GPs were the predominant type of health professional (90\%) referring clients to PMHC services, and nearly a quarter of clients had previously commenced at least one service session related to the same or different diagnostic conditions to PMHC services (table 1).

Multivariable analysis indicated poor proficiency of English compared with those who speak English very well $(\mathrm{OR}=1.64,95 \%$ CI: 1.23 to 2.20$)$, those who resided in the lowest SES areas compared with the highest SES quintile (OR=1.57, 95\% CI: 1.34 to 1.83$)$, and those aged 25-44 $(\mathrm{OR}=1.63,95 \%$ CI: 1.26 to 2.11$), 12-24(\mathrm{OR}=1.92,95 \%$ CI: $1.47-2.49)$ and $<=11(\mathrm{OR}=1.69,95 \% \mathrm{CI}=1.26-2.28)$, compared with the older age group (age $>=65$ ), were more likely to not attend treatment sessions. Those who spoke a language other than English were less likely to not attend first psychological treatments $(\mathrm{OR}=0.65,95 \%$ $\mathrm{CI}=0.51-0.84)$. In addition, those with substance use disorders compared with anxiety disorders $(\mathrm{OR}=1.55$, $95 \% \mathrm{CI}=1.17,2.06)$, and those use psychotropic medication at the time of referral (OR=1.20,95\% CI=1.06-1.36) were more likely to not attend treatments, while those with a risk of suicide were less likely to not attend treatments $(\mathrm{OR}=0.70,95 \% \mathrm{CI}=0.59-0.84)$. Those referred by a social health worker $(\mathrm{OR}=2.04,95 \%$ CI: 1.36 to 3.05$)$, other professionals (OR=1.72, 95\% CI: 1.30 to 2.29) or a mental health nurse (OR=1.49, $95 \%$ CI: 1.03 to 2.16$)$ compared with a referral by a GP were more likely to not attend psychological treatments, whereas those who previously used PMHC services $(\mathrm{OR}=0.66,95 \%$ CI: 0.58 to 0.76 ) were less likely to not attend to the first treatment session. Gender, marital status and employment status were not significantly associated with treatment nonattendance (table 2; model 4 and online supplemental tables 1,2; model 4 and figure 1).

Similar associations were evident in age-specific analyses for younger (age $\leq 24$ years), and older (age $>24$ years) age groups (table 2 and online supplemental tables 1 and 2). Among the older age-group ( $>24$ years) individuals at risk of suicide, and those with poor English proficiency, were more likely to not attend first treatment session compared with the younger age cohort (aged $\leq 24$ years) (online supplemental table 2). In contrast, the younger aged cohort (aged $\leq 24$ years) who did not attend their first treatment session were more likely to be using psychotropic medications, present with substance abuse, and be referred by a social worker or a mental health nurse, compared with older aged cohorts (aged >24 years) (online supplemental table 1).

\section{DISCUSSION}

The current study investigated the determinants of first treatment session non-attendance among those referred to PMHC services in Western Sydney (Australia). Overall, 19.3\% of referrals did not result in subsequent service attendance, a percentage within the range $(16 \%-48 \%)$ of similar studies investigating first treatment session non-attendance, ${ }^{5} 6121323$ and slightly lower compared with the national ATAPS figure of $21 \%$ based on data between 2003 and 2012. ${ }^{24}$ 
Open access

Table 2 Generalized estimating equation models to predict first treatment session non-attendance

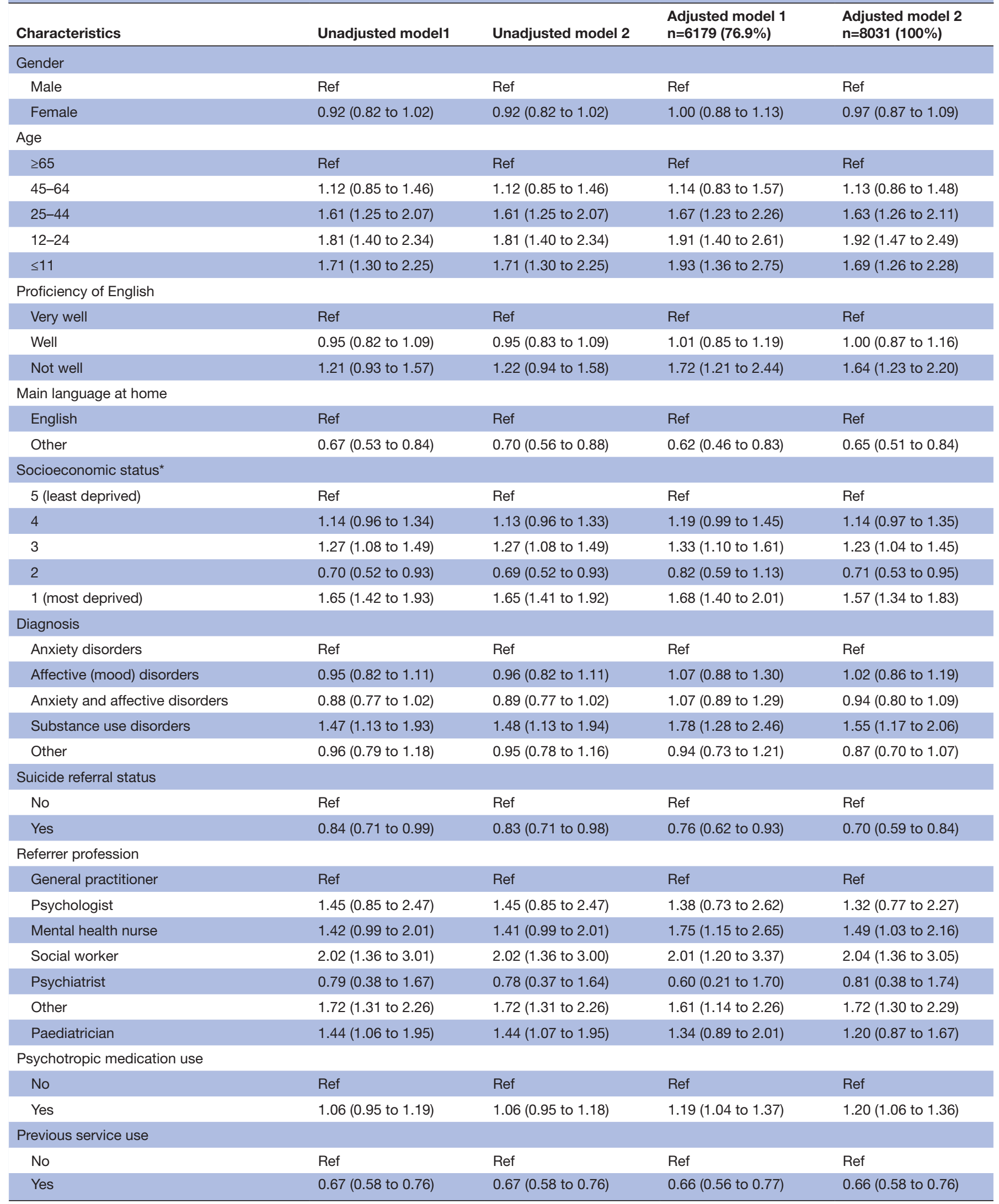

ORs and $95 \%$ Cls were presented as effects; $n$-number of clients; Unadjusted model1 - univariate model based on non-imputed data; Unadjusted model2univariate model based on imputed data; Adjusted model1 - multivariate model based on non-imputed data; Adjusted model2 - multivariate model based on imputed data.

*Area-level socioeconomic status based on Index of Relative Socio-economic Advantage and Disadvantage. 


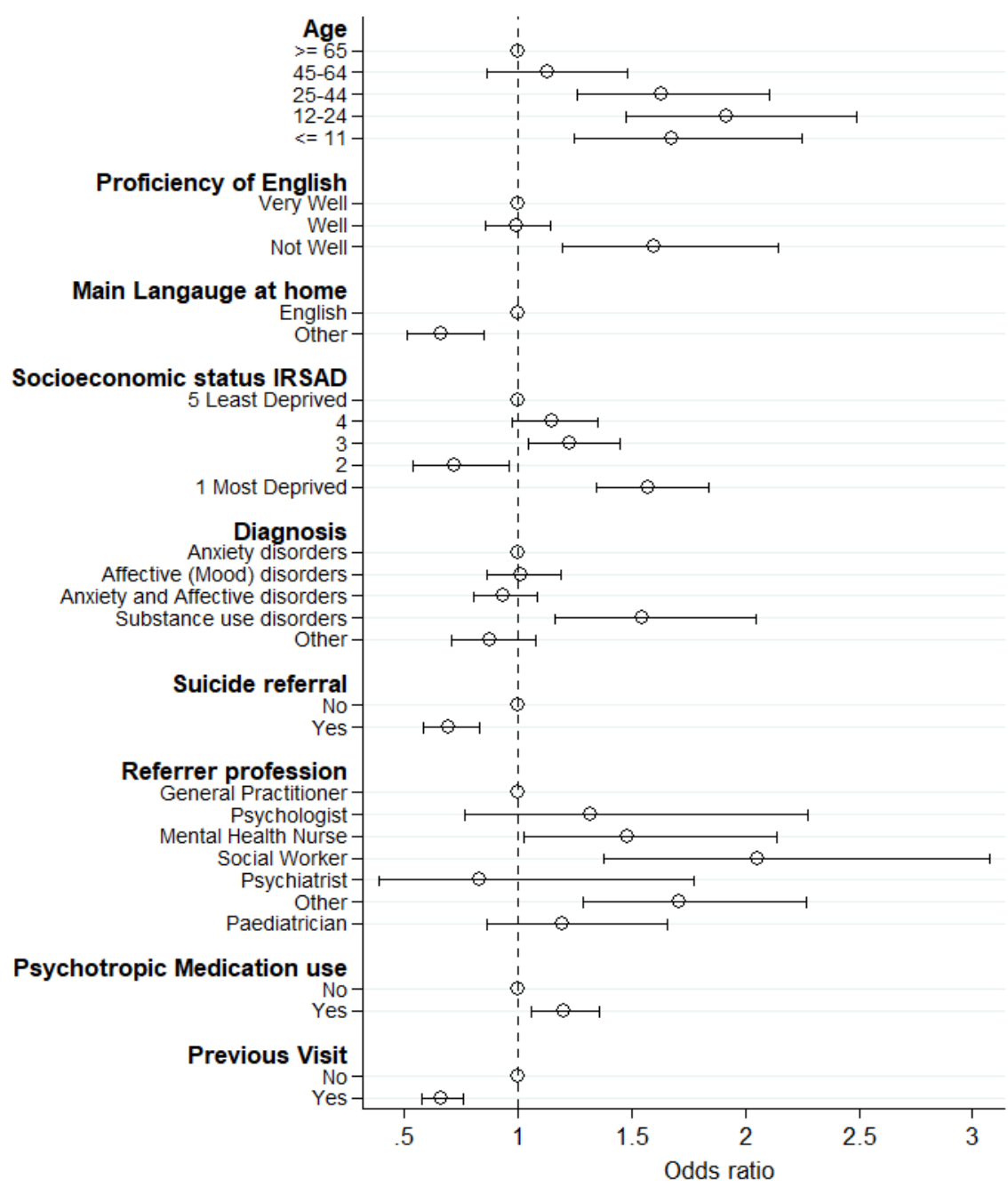

Figure 1 ORs and $95 \%$ Cls of covariates predicting first treatment non-attendance. Note: The $95 \% \mathrm{Cl}$ for a covariate category not crossing the vertical dash line with $\mathrm{OR}=1$ indicates that the OR for that category is significantly different from 1 , and vice versa. IRSAD, Index of Relative Socio-economic Advantage and Disadvantage.

Younger age groups (age <45) were more likely to not attend follow-up treatment sessions compared with older age cohorts (age $\geq 45$ ), which was also consistent with previous similar studies. ${ }^{512}$ A higher likelihood of treatment nonattendance among younger age groups may reflect negative perceptions about the mental health support they receive, and a greater reliance on support from peers. ${ }^{25}$ It is also possible, especially among children aged 5-11 years, that support from parents or guardians in facilitating treatment access may not be sufficient. ${ }^{26}$

Gender and marital status were not consistently associated with treatment non-attendance in the present study. Previous studies also found inconsistent associations between these sociodemographic factors and first treatment session nonattendance to mental health services. Di Bona $e t$ al found gender was not associated with treatment non-attendance, ${ }^{13}$ whereas Simon and Ludman found females were more likely to not attend first treatment session. ${ }^{12}$ Similarly, Lester and Harris found those who were divorced were more likely to not attend a first treatment session ${ }^{27}$ and Matas et al found that those who were single were more likely to not attend the first treatment session, ${ }^{10}$ whereas Cheng et al. and Fenger et al found no association between marital status and first treatment session non-attendance. ${ }^{25}$ In the present study, univariate analyses found those who were divorced were more likely to attend treatments compared with those married, never married or separated, but associations were not strong following adjustment for other covariates.

Employment status was not associated with treatment nonattendance in the present study, which was also consistent with a previous similar study in Denmark ${ }^{5}$ which showed no association for first treatment non-attendance, but did find that unemployment was associated with treatment dropout over subsequent sessions. In contrast, the present study found that those from lower SES areas were more likely to not attend the first treatment session compared with those residing in higher SES areas, except the second lowest quintile of SES but the effect on treatment non-attendance after combining the lowest and second lowest quintiles was significantly greater compared with the highest SES quintile ((OR=1.39, 95\% CI: 1.20 to 1.62); this result was not shown in tables). This may, however, reflect differences in the level 
of mental health service availability, the material resources among those residing in lower SES areas and the location and distance to travel for services. ${ }^{28} 29$

Interestingly, individuals who spoke languages other than English were more likely to attend treatments compared with English language speakers, whereas individuals with a poor English proficiency (perhaps more common among those with a non-English speaking background) were more likely to not attend first treatment session. A possible interpretation of this finding may reflect duration of residence in Australia in migrant communities in Western Sydney (a variable not available on the PMHC dataset), and recent migrants within these communities may have poorer English language proficiency that may present a barrier to initially accessing these mental health services. However, there are established culturally and linguistically diverse communities in Western Sydney (predominantly Indian, Chinese, Filipinos and Sri Lankan) ${ }^{17}$, with corresponding mental health services that can be provided by professionals of similar cultural backgrounds.

Those presenting with a (comorbid) substance use disorder were less likely to attend subsequent treatments. Substance use disorders present additional complexity to remain in treatment when comorbid with other conditions, ${ }^{1}$ but behavioural engagement strategies may have the potential to increase treatment engagement. ${ }^{30}$ In contrast, those assessed to be at risk of suicide were more likely to attend treatment services, which differs from previous studies suggesting that those at risk of suicide were less likely to attend follow-up treatments, ${ }^{13} 31$ and may reflect the current PHN requirements to provide immediate access (within 7 days of the date of referral) to treatments for those with risk of suicide. ${ }^{32}$

In comparison, those who were newly referred to PMHC services, and those using psychotropic medication at the time of referral were more likely not to attend follow-up treatment sessions. The latter findings suggest that there may be perceptions that psychological treatments in combination with psychotropic medication are not helpful. Additionally, clients who were referred by a source other than a GP had higher non-attendance rates compared with those referred by a GP and this may likely reflect established relationships between clients and an existing GP, and different referral processes and levels of existing communication between GPs and specific PMHC services than for other non-GP referrers.

There are a number of methodological limitations when interpreting findings from the present study. First, the current study was unable to include a number of factors that may be important determinants of treatment non-attendance. Level of psychological distress or severity of illness, ${ }^{33} 34$ waiting time between the initial presentation and the first follow-up service $^{15}$ and transportation barriers or travel distance ${ }^{27} 35$ have previously been shown to be associated with treatment non-attendance. The PMHC MDS, however, did not collect information on these factors. Baseline psychological distress (as a proxy measure of illness severity) measured by the Kessler 10 (K10) Score is collected in both ATAPS and PHMC MDSs, but it is not entered by service providers with much consistency (K10 scores were missing on $~ 83 \%$ of referrals during the study period). However, findings from the current study are generally consistent with the findings of previous research, and it is unlikely that estimates would substantially change in the presence of these unmeasured covariates. Second, it is possible that those individuals who did not attend any treatment sessions may have accessed other fee for service mental health services not captured by the PMHC MDS. This would represent a small proportion of all services delivered, given the PMHC services described are provided at a no cost (or low cost). Thirdly, exclusion of low intensity interventions may reduce the generalisability of findings to those at risk or experiencing mild mental health conditions, in that the services captured in the current study would likely reflect slightly more severe cases than mild conditions of mental disorders who may be less likely to engage and attend mental health interventions. ${ }^{36}$

\section{CONCLUSION}

Findings of the current research can help to identify vulnerable groups at risk of treatment non-attendance at the time they are being referred to PMHC services, and can inform local policy makers in the development of relevant policies and guidelines to enhance the delivery of PMHC services. Youth-specific treatment approaches that aim to improve attendance and follow-up, and behavioural engagement strategies, such as, motivational interviewing techniques especially for those experiencing substance use disorders, are important areas for future enquiry. Additionally, for those of culturally and linguistically diverse backgrounds the provision of treatment services with a provider of similar cultural background, and improvements in capacity for mental health training arrangements among allied health professionals are areas of focus for primary care service and policy responses to improve mental health outcomes in Western Sydney.

Contributors SM conceptualised the study, analysed the data and wrote the first draft of the manuscript. AP, HM, SF and BP provided advice on the study conception. $\mathrm{AP}, \mathrm{HM}, \mathrm{SF}$ and $\mathrm{BP}$ contributed to the analysis. AP contributed to critically revise the first draft and provided overall supervision and guidance for the manuscript. HM, SF and BP made suggestions and revisions to the manuscript. All authors read and approved the final manuscript.

Funding The authors have not declared a specific grant for this research from any funding agency in the public, commercial or not-for-profit sectors.

Competing interests None declared.

Patient consent for publication Not required.

Ethics approval The data described under this study were accessed in accordance with approvals by the Western Sydney University Human Research Ethics Committee (Approval No.H13040).

Provenance and peer review Not commissioned; externally peer reviewed.

Data availability statement Data may be obtained from a third party and are not publicly available. The primary mental health care (PMHC) Minimum Data Set (MDS) and Access To Allied Psychological Services MDS are owned by the Australian Government Department of Health. Western Sydney Primary Health Network (PHN) has access to this data for Western Sydney PHN's population catchment area. Data model, variables and variable labels of PMHC MDS can be found via https://docs. pmhc-mds.com/projects/data-specification/en/v1/data-model-and-specifications. html\#data-model.

Supplemental material This content has been supplied by the author(s). It has not been vetted by BMJ Publishing Group Limited (BMJ) and may not have been peer-reviewed. Any opinions or recommendations discussed are solely those of the author(s) and are not endorsed by BMJ. BMJ disclaims all liability and 
responsibility arising from any reliance placed on the content. Where the content includes any translated material, BMJ does not warrant the accuracy and reliability of the translations (including but not limited to local regulations, clinical guidelines, terminology, drug names and drug dosages), and is not responsible for any error and/or omissions arising from translation and adaptation or otherwise.

Open access This is an open access article distributed in accordance with the Creative Commons Attribution Non Commercial (CC BY-NC 4.0) license, which permits others to distribute, remix, adapt, build upon this work non-commercially, and license their derivative works on different terms, provided the original work is properly cited, appropriate credit is given, any changes made indicated, and the use is non-commercial. See: http://creativecommons.org/licenses/by-nc/4.0/.

ORCID iD

Sithum Munasinghe http://orcid.org/0000-0003-3650-1889

\section{REFERENCES}

1 Mitchell AJ, Selmes T. Why don't patients attend their appointments? Maintaining engagement with psychiatric services. Advances in Psychiatric Treatment 2007;13:423-34.

2 Cheng K-D, Huang C-J, Tsang H-Y, et al. Factors related to missed first appointments after discharge among patients with schizophrenia in Taiwan. J Formos Med Assoc 2014;113:436-41.

3 Page A, Atkinson J-A, Campos W, et al. A decision support tool to inform local suicide prevention activity in greater western Sydney (Australia). Aust N Z J Psychiatry 2018;52:983-93.

4 Moore CG, Wilson-Witherspoon P, Probst JC. Time and money: effects of no-shows at a family practice residency clinic. Family Medicine-Kansas City 2001;33:522-7.

5 Fenger M, Mortensen EL, Poulsen S, et al. No-shows, Drop-outs and completers in psychotherapeutic treatment: demographic and clinical predictors in a large sample of non-psychotic patients. Nord J Psychiatry 2011;65:183-91.

6 Mitchell AJ, Selmes T. A comparative survey of missed initial and follow-up appointments to psychiatric specialties in the United Kingdom. Psychiatr Serv 2007;58:868-71.

7 Barrett MS, Chua W-J, Crits-Christoph P, et al. Early withdrawal from mental health treatment: implications for psychotherapy practice. Psychotherapy 2008;45:247-67.

8 Simon GE, Imel ZE, Ludman EJ, et al. Is dropout after a first psychotherapy visit always a bad outcome? Psychiatr Serv 2012;63:705-7.

9 Kruse GR, Rohland BM. Factors associated with attendance at a first appointment after discharge from a psychiatric hospital. Psychiatr Serv 2002;53:473-6.

10 Matas M, Staley D, Griffin W. A profile of the noncompliant patient: a thirty-month review of outpatient psychiatry referrals. Gen Hosp Psychiatry 1992;14:124-30.

11 Self R, Oates P, Pinnock-Hamilton T, et al. The relationship between social deprivation and unilateral termination (attrition) from psychotherapy at various stages of the health care pathway. Psychol Psychother 2005;78:95-111.

12 Simon GE, Ludman EJ. Predictors of early dropout from psychotherapy for depression in community practice. Psychiatr Serv 2010;61:684-9.

13 Di Bona L, Saxon D, Barkham M, et al. Predictors of patient nonattendance at improving access to psychological therapy services demonstration sites. J Affect Disord 2014;169:157-64.

14 Marshall D, Quinn C, Child S, et al. What IAPT services can learn from those who do not attend. J Ment Health 2016;25:410-5.

15 Reitzel LR, Stellrecht NE, Gordon KH, et al. Does time between application and case assignment predict therapy attendance or premature termination in outpatients? Psychol Serv 2006;3:51-60.
16 National Mental Health Commission. The National review of mental health programmes and services. 67. Sydney: NMHC, 2014.

17 PHIDU. Social health atlas of Australia: primary health networks, 2019. Available: http://phidu.torrens.edu.au/social-health-atlases/ data\#social-health-atlas-of-australia-primary-health-networks [Accessed 10 Nov 2019].

18 WentWest Primary Health Network. Phn needs assessment western Sydney primary health Netork 2019-2022. Available: http://www. wentwest.com.au/documents/phn/programs/capacity-capability/ Reports_WSPHN_Needs-Assessment_201921.pdf [Accessed 12 Jul 2019].

19 Hickie I, Groom G. Primary care-led mental health service reform: an outline of the better outcomes in mental health care initiative. Australasian Psychiatry 2002;10:376-82.

20 Australian Government Department of Health and Ageing Operational guidelines for the access to allied psychological services initiative, 2012. Available: https://www.aasw.asn.au/document/ version/5241 [Accessed 2 Sep 2019].

21 Australian Bureau of Statistics. Socio-Economic indexes for areas, 2018. Available: https://www.abs.gov.au/websitedbs/censushome. nsf/home/seifa [Accessed 10 Apr 2019].

22 Buuren S, Groothuis-Oudshoorn K. Mice: multivariate imputation by chained equations in R. J Stat Softw 2010:1-68.

23 Issakidis C, Andrews G. Pretreatment attrition and dropout in an outpatient clinic for anxiety disorders. Acta Psychiatr Scand 2004;109:426-33.

24 Bassilios B, Nicholas A, Reifels L, et al. Achievements of the Australian access to allied psychological services (ATAPS) program: summarising (almost) a decade of key evaluation data. Int J Ment Health Syst 2016;10:61.

25 Rickwood DJ, Deane FP, Wilson CJ. When and how do young people seek professional help for mental health problems? Med J Aust 2007;187:S35-9.

26 Nock MK, Ferriter C. Parent management of attendance and adherence in child and adolescent therapy: a conceptual and empirical review. Clin Child Fam Psychol Rev 2005;8:149-66.

27 Lester S, Harris SM. Factors associated with first session nonattendance at a university-based family therapy clinic. Am J Fam Ther 2007;35:363-76.

28 Packness A, Waldorff FB, Christensen RdePont, et al. Impact of socioeconomic position and distance on mental health care utilization: a nationwide Danish follow-up study. Soc Psychiatry Psychiatr Epidemiol 2017;52:1405-13.

29 Zimmermann D, Rubel J, Page AC, et al. Therapist effects on and predictors of Non-Consensual dropout in psychotherapy. Clin Psychol Psychother 2017;24:312-21.

30 Molfenter T. Reducing appointment no-shows: going from theory to practice. Subst Use Misuse 2013;48:743-9.

31 Zivin K, Pfeiffer PN, McCammon RJ, et al. "No-shows": who fails to follow up with initial behavioral health treatment? Am J Manag Care 2009;15:105-12.

32 Department of Health. Phn program performance and quality framework., 2018. Available: https://www1.health.gov.au/ internet/main/publishing.nsf/Content/55B22FCB1BB6A94E CA257F14008364CC/\$File/V1.1\%20-\%20PHN\%20Program\% 20Performance\%20and\%20Quality\%20Framework.pdf. [Accessed 12 Jul 2019].

33 Al-Asadi AM, Klein B, Meyer D. Pretreatment attrition and formal withdrawal during treatment and their predictors: an exploratory study of the anxiety online data. J Med Internet Res 2014;16:e152.

34 Killaspy H, Banerjee S, King M, et al. Prospective controlled study of psychiatric out-patient non-attendance. characteristics and outcome. Br J Psychiatry 2000;176:160-5.

35 Long J, Sakauye K, Chisty K, et al. The empty chair appointment. Sage Open 2016;6:215824401562509.

36 Gyani A, Shafran R, Layard R, et al. Enhancing recovery rates in IAPT services: lessons from analysis of the year one data, 2011. 